\title{
CLINICAL FEATURES AND OUTCOME OF CHILDREN ADMITTED WITH HERBAL INTOXICATION AT SAHLOUL UNIVERSITY HOSPITAL, SOUSSE, TUNISIA
}

\section{H. Ajmi, M. Barka, S. Mabrouk, F. Majdoub, J. Chemli, N. Zouari, S. Ghorbel, S. Hassayoun, S. Abroug.}

\section{Sahloul University Hospital, Pediatric deparment, Sousse, Tunisia}

Background and Aims:

In the past, drugs and caustics were the mainly forms of poisonings in children. Lately, we are witnessing the emergence of new kinds of intoxication: "herbal intoxications". Besides their therapeutic potency, some plants have strong toxicity among human, especially children.

The aim of our study was to analyze the clinical patterns of poisonous plants intoxications, its management strategies and its outcomes in pediatric patients.

Method:

Retrospective chart review of patients admitted for plants poisoning, to the pediatric ward of the University Hospital of Sahloul, Sousse between January 2000 and December 2017.

Results:

A total of nine children were included in our study. The mean age at admission was 11 years old [6-15 years old] and the sex ratio was of o.8. Physical examination revealed an impairment of consciousness in 6 cases (66\%), a disturbance of behavior in 2 cases (22\%), bilateral areflective mydriasis in 7 children (77\%) and a hematoma of the calf muscle in one case. Table 1 summarize the clinical features of each patient.

Table 1: clinical features of patients with herbal intoxication

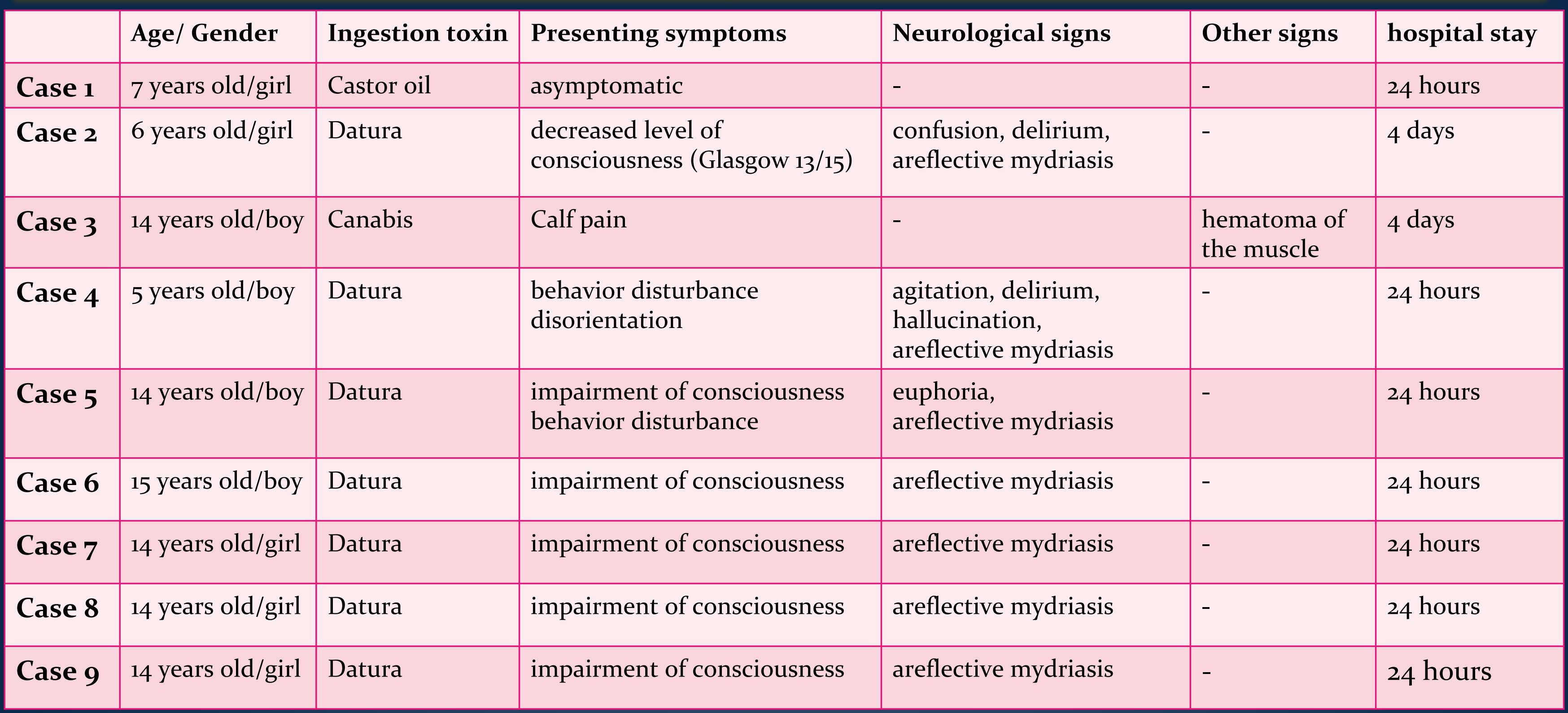

The poisoning was voluntary in 6 cases: 5 patients were involved in a group intoxication out of curiosity, to have fun. One patient had a regular consumption of canabis, linked to dependency and family problems.

For the other cases, it was an accidental poisoning.

The ingestion of Datura was the most frequent cause of intoxication: 7 cases (77\%).

Only one patient was hospitalized in pediatric intensive care unit , no one required

intubation and ventilatory support.

Treatment of patients consists on gastric lavage with saline solution in 1 case (11\%),

oral activated charcoal $(\mathrm{gg} / \mathrm{kg})$ and forced diuresis $(3 \mathrm{~L} / \mathrm{m} 2)$ in 7 cases $(77 \%)$.

All patients had a good recovery after treatment with an average hospital stay of 40 hours."

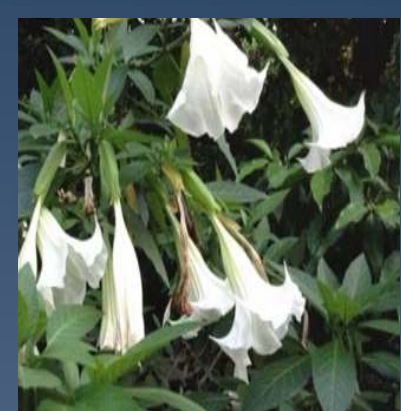

Discussion:

According to poison control centers, children poisoning with plants represents of 2 to $2.5 \%$, most often affecting children aged 1 to 4 years old in $90 \%$ of cases. Datura stramonium has become quite popular and the number of hospitalizations for acute datura poisoning has increased rapidly. While many herbal products are innocuous or possess minimal toxicity, some contain toxic ingredients that may have dangerous side effects, it can even cause death. The symptoms of acute poisoning differ:

$>$ Digestive with nausea, abdominal pain, vomiting, significant diarrhea and dehydration, or even irritation, edema of the mouth and pharynx with respiratory difficulties (Diffenbachia, Euphorbia) and hepatotoxicity (castor oil).

Atropinic syndrome, rare (Belladonna, Datura, Jusquiame)

Neurological: seizures (Oenanthe safrane, Jequiry), decreased level of consciousness, confusion, incoherent speech, visual disturbances, hearing and visual hallucinations, coma.

Most of the cases described in the literature had a good prognosis after supportive treatment. Currently, voluntary plant poisoning increases in young adolescents because of their accessibility. Teenagers voluntarily ingest the plant seeking its natural hallucinogenic and euphoric effects and represent most of the cases reported in the literature. It can even cause dependance.

Every acute anticholinergic syndrome presenting with coma/seizures, in young people lacking other objective findings, can suggest poisoning with plants especially Datura stramonium, and early decontamination/management should be initiated.

Since supportive therapy is the only therapeutic approach for the treatment of acute intoxication with plant, and there is no specific therapy for most plant poisonings, medicinal plants should be used with caution. 